\title{
Evolution of the Magnetic Field of a Neutron Star During Polar-Cap Accretion
}

\author{
A. Melatos \\ Department of Astronomy, 601 Campbell Hall, University of California \\ at Berkeley, Berkeley CA 94720 USA
}

E. S. Phinney

Theoretical Astrophysics, Mail Code 130-33, California Institute of Technology, Pasadena CA 91125 USA

\section{Introduction}

Neutron stars that have experienced episodes of mass transfer exhibit lower magnetic dipole moments $\mu$ than other neutron stars, e.g. binary radio pulsars with high-mass $\left(\mu \sim 10^{28} \mathrm{Gcm}^{3}\right)$ and low-mass $\left(\mu \sim 10^{27} \mathrm{G} \mathrm{cm}^{3}\right)$ companions, isolated millisecond pulsars $\left(\mu \sim 10^{26} \mathrm{G} \mathrm{cm}^{3}\right)$, and accreting objects in low-mass $\mathrm{X}$-ray binaries $\left(\mu<10^{27} \mathrm{G} \mathrm{cm}^{3}\right)$. Indeed, the $\mu$-versus-orbital-period and $\mu$ versus-age relations for low-mass and high-mass binaries imply that $\mu$ decreases monotonically with accreted mass $M_{\mathrm{a}}$ (Taam \& van den Heuvel 1986; van den Heuvel \& Bitzaraki 1995). Simple Ohmic field decay cannot be responsible given the existence of cold and therefore old white-dwarf companions (Kulkarni 1986). Alternative mechanisms include accretion-induced heating of the crust (Konar \& Bhattacharya 1997) and fluxoid-vortex interactions in the stellar interior (Srinivasan et al. 1990).

\section{Equatorward Hydromagnetic Spreading}

An accretion flow channeled onto the magnetic poles of a neutron star reduces $\mu$ without dissipation by squeezing the magnetic field into a narrow, flaring equatorial belt or 'tutu', thereby enhancing higher-order multipoles at the expense of the dipole (Melatos \& Phinney 1999). During the early stages of accretion, material accumulates in a magnetically confined hillock on the polar cap (Brown $\&$ Bildsten 1998). Once $M_{\mathrm{a}}$ exceeds $\sim 10^{-5} M_{\odot}$, the hydrostatic pressure gradient at the base of the hillock exceeds the local magnetic tension force, the field lines buckle, and the base of the hillock spreads laterally towards the equator, dragging frozen-in polar magnetic flux along with it and compressing it until a new equilibrium is established.

The hydromagnetic structure of a neutron star accreting at its poles can be calculated as a function of $M_{\mathrm{a}}$ by starting from a spherical Lane-Emden polytrope with a central, point-like, magnetic dipole and letting the configuration evolve through a quasi-static sequence of two-dimensional, Grad-Shafranov (i.e. ideal-MHD) equilibria (Uchida \& Low 1981; Melatos \& Phinney 1999; cf. dissipative, spherically symmetric analysis of Romani 1990). The resulting $\mu$-versus- $M_{\mathrm{a}}$ 


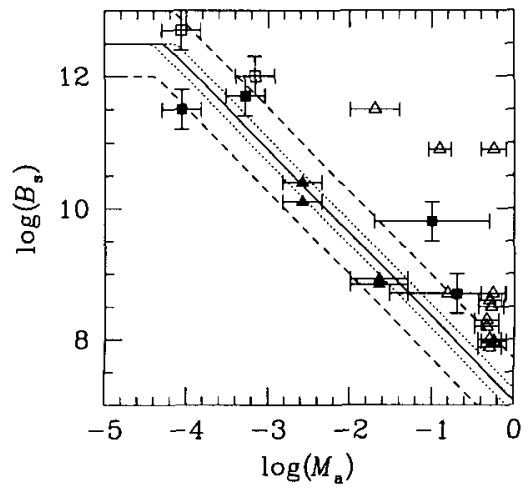

Figure 1. Surface dipole field $B_{\mathrm{s}}$ (in $\mathrm{G}$ ) versus accreted mass $M_{\mathrm{a}}$ (in $M_{\odot}$ ) for various neutron-star binaries. Data: He white dwarf (open $\triangle$ ), CO white dwarf or double neutron star (filled $\triangle$ ), HMXB (open box), LMXB (filled box). Theory: $\log \left(B_{\mathrm{s} 0} / \mathrm{G}, \dot{M}_{\mathrm{a}} / M_{\odot} \mathrm{yr}^{-1}\right)=(12.5,-8)$ (solid), (12.5, $-8 \pm 1$ ) (dotted), $(12.5 \pm 1,-8)$ (dashed).

relation (Fig. 1) agrees well with observations. The few outliers are peculiar in other respects too, e.g. very-low-mass companions, large orbital eccentricities.

\section{Gravitational Radiation}

Hydromagnetic stresses in the equatorial magnetic tutu create a conjugate material distortion that radiates gravitational waves. The fractional difference between the principal moments of inertia, computed from the Grad-Shafranov equilibria described above, is given by $\epsilon \approx 2 \times 10^{-5}\left(M_{\mathrm{a}} / M_{\odot}\right)^{1.7}$ (Melatos \& Phinney 1999). Hence gravitational waves from isolated millisecond pulsars and pulsars in He-white-dwarf binaries with $M_{\mathrm{a}} \sim 0.1 M_{\odot}$ ought to be detectable by interferometers under construction, e.g. LIGO. Combining $\epsilon\left(M_{\mathrm{a}}\right)$ with $\mu\left(M_{\mathrm{a}}\right)$ yields a testable, inverse correlation between wave amplitude and $\mu$.

\section{References}

Brown E. F., Bildsten L., 1998, ApJ, 496, 915

Konar S., Bhattacharya D., 1997, MNRAS, 284, 311

Kulkarni S. R., 1986, ApJ, 306, L85

Melatos A., Phinney E. S., 1999, ApJ, submitted

Romani R. W., 1990, Nature, 347, 741

Srinivasan G., Bhattacharya D., Muslimov A., Tsygan A. 1990, Curr. Sci., 59, 31

Taam R. E., van den Heuvel E. P. J., 1986, ApJ, 305, 235

Uchida Y., Low B. C., 1981, J. Astrophys. Ast., 2, 405

van den Heuvel E. P. J., Bitzaraki O., 1995, A\&A, 297, L41 